\title{
Metal Oxide Nanowire Gas Sensors
}

\author{
Francisco Hernandez-Ramirez ${ }^{1,2, *}$, Juan Daniel Prades ${ }^{1}$ \\ and Joan Ramon Morante ${ }^{1}$ \\ ${ }^{1}$ Catalonia Institute for Energy Research (IREC), \\ Josep Pla, 2. B3, Ground Floor, E-0819 Barcelona, Spain \\ ${ }^{2}$ Electronic Nanosystems, S. L. (e-Nanos), \\ Baldiri Reixac, 4-8, Parc Cientific. Torre D, E-08028 Barcelona, Spain
}

(Received April 22, 2009; accepted May 11, 2009)

Key words: metal oxide, nanowire, gas, sensor

Monocrystalline metal oxide nanowires have emerged as new building blocks for a wide range of devices and applications owing to their unique properties directly derived from their size, crystalline structure and well-defined geometry. In particular, these nanomaterials have been successfully integrated in proof-of-concept gas sensors with high sensitivity and stable characteristics, giving rise to a productive research line in recent years. In this article, we describe the state-of-the-art research and development activities in metal oxide nanowire gas sensors, and survey the main characteristics of these devices in terms of response, stability and selectivity towards gases. Finally, some predictions of future developments in metal oxide nanowire gas sensors are presented and briefly discussed.

\section{Introduction}

One-dimensional semiconductor nanowires have attracted considerable research interest during recent years owing to their novel electrical, magnetic and chemical properties, and their potential to build new systems for future nanoscale electronics. ${ }^{(1-3)}$ Many semiconductor materials have been made into single-crystal nanowires with superior structural quality and high mobility. Among them, metal oxides are particularly interesting because they are the foundation of many devices, such as gas sensors, photodetectors and varistors. ${ }^{(1,2)}$ To date, significant progress has been made in the synthesis and characterization of nanowires and their integration into proof-of-concept systems. ${ }^{(4-8)}$ Nevertheless, all these works are still in a preliminary stage of development, and some fundamentals remain to be clarified. ${ }^{(9)}$

The wish to make good use of the unique properties of monocrystalline metal oxide nanowires, such as the high surface-to-volume ratio and the well-defined geometry, has lead to intensive research efforts to obtain a new generation of gas sensors with improved performance and to shed some light on the typical nanoscale phenomena.

${ }^{*}$ Corresponding author: e-mail: fhernandezra@gmail.com 
Here, state-of-the-art gas sensors based on metal oxide nanowires are presented, and their responses towards gases are studied in terms of the selectivity, sensitivity and stability of the analyzed prototypes. Nanowire-based technologies are still not mature enough to become competitive in relation to standard and commercial technologies. For this reason, the main aims of this study are simply to survey the field of research on metal oxides, which has been extremely active over the last few years, and identify the main advantages and drawbacks of using nanowires as building blocks of innovative gas sensors.

\section{Discussion}

Metal oxide gas microsensors have become a success story in the last few decades, as demonstrated by the large number of companies currently releasing these products on the market. ${ }^{(10,11)}$ Commercial devices typically consist of a thin layer of a selected metal oxide deposited onto hotplates, which are used to heat the layer and thus activate the interaction between the sensing layer and the gas molecules. This chemicoelectrical transduction mechanism leads to a substantial modulation of the device's electrical resistance, which is easily monitored in conductometric measurements. ${ }^{(10,12)}$ Although this technology is mature, it presents unresolved drawbacks such as the lack of selectivity, high power consumption and poor stability. ${ }^{(10,12)}$ Consequently, metal oxide sensors are at a disadvantage in relation to other well-established technologies (i.e., infrared IR sensors, electrochemical cells). ${ }^{(10)}$

In recent years, intensive research efforts have been carried out with the aim of developing breakthrough metal oxide-based technologies capable of providing higher sensitivity and selectivity with reduced cost and power consumption, exploring novel concepts and intensively using nanomaterials. ${ }^{(2,7,13-16)}$ In particular, metal oxide nanowires have several advantages over film sensors owing to their geometry and physical, chemical and electronic properties. ${ }^{(1,2,6)}$ The high surface-to-volume ratio of quasi-one-dimensional (Q1D) nanostructures guarantees that most of the atoms are available for surface reactions with gas molecules, giving rise to a strong effect of surface processes on the electronic properties. Furthermore, their superior stability because of the high crystallinity and the possibility of attaining large-scale production following simple and well-controlled synthesis methods have turned metal oxide nanowires into excellent building blocks of a new generation of devices with promising performances (Fig. 1). Here, the advantages and disadvantages of using metal oxide nanowires as gassensing elements in terms of response, stability and selectivity are reviewed.

\subsection{Sensor response}

The response to gases of individual nanowires is directly related to the modulation of the electrical conduction along them owing to the presence of gas molecules in air. ${ }^{(17)}$ This effect is based on the capture and release of electrical charges by adsorbed species, which modifies the nanowire's electrical resistance $R_{\mathrm{NW}}$. According to this theoretical description, 


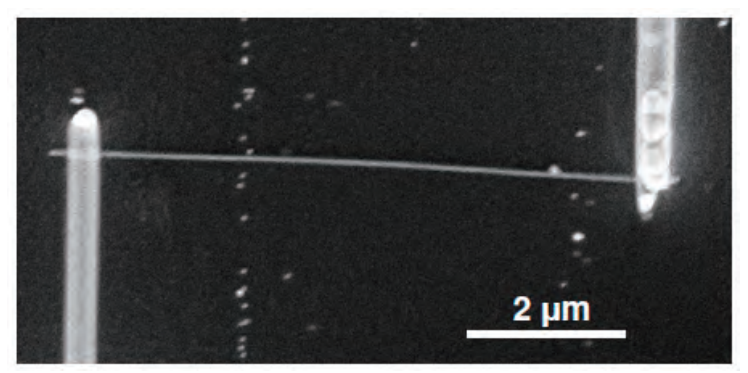

Fig. 1. Metal oxide nanowire electrically contacted by FIB lithography. A detailed explanation of its properties and the fabrication process were published elsewhere. ${ }^{(4)}$

the dependence of $R_{\mathrm{NW}}$ as a function of the gas concentration can be written as ${ }^{(17,18)}$

$$
R_{\mathrm{NW}}=\frac{\rho L}{\pi(r-\lambda)^{2}}
$$

where $\rho$ is the nanowire resistivity, $L$ is the nanowire length, $r$ is the nanowire radius and $\lambda$ is the width of the depletion layer created by adsorbed molecules (Fig. 2). Here, it should be pointed out that $\lambda$ is a characteristic function for a given gas species that depends on the molecular concentration in air as well as the intrinsic electrical parameters of each metal oxide nanowire. Equation (1) establishes a direct connection between the nanowire radius and the gas response $S$; the thinner the nanowires, the higher the response $S$ (Fig. 3). ${ }^{(17)}$

The study of individual nanowires simplifies the experimental scenario typical of conventional metal oxide sensors, which consist of porous films formed by randomly agglomerated nanoparticles, ${ }^{(10,12)}$ and provides a straightforward methodology to design high-response sensors; ultrathin nanowires (radii below $40 \mathrm{~nm}$ ) are required to maximize the response $S$ of these prototypes (Fig. 3). ${ }^{(9,17)}$ Although the same relationship between the size of nanoparticles in thin-film sensors and the device response has been reported in the past, ${ }^{(10,12,19)}$ the use of a single nanowire to obtain metal oxide sensors minimizes interfering effects, which have a strong effect on $S$ in thin-film based devices, such as the sensing layer agglomeration and the uncontrolled characteristics of necks and boundaries among nanoparticles. ${ }^{(9)}$ The same conclusions are reached if bundles of nanowires are used as a sensing layer. For this reason, the use of individual nanowires to build up gas sensors is extremely advisable if the sensor response $S$ needs to be accurately controlled.

Nanowire-based sensors still exhibit worse performance in terms of $S$ than their micro-counterparts. Nevertheless, both technologies are not at the same stage of development, which makes it difficult to compare them. On one hand, the study and optimization of thin-film-based sensors have been carried out for many years, ${ }^{(10)}$ giving rise to manifold strategies to optimize their design, and thus improve their performance. 


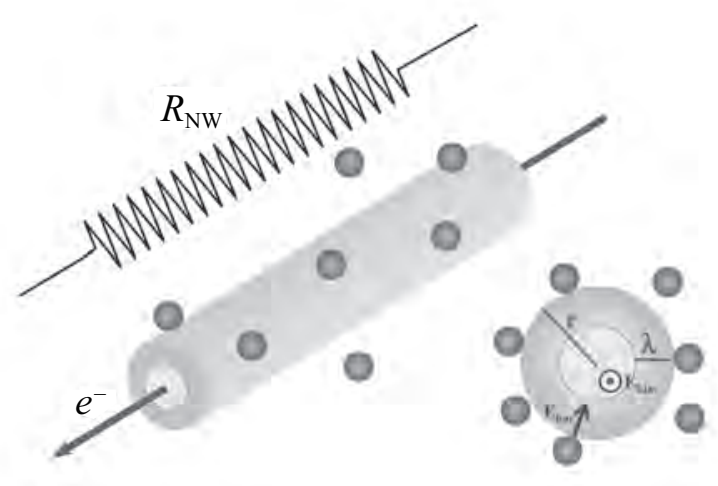

Fig. 2. Diagram of the gas sensing mechanism in metal oxide nanowires. Adsorbed molecules at the surface modify the depletion layer $\lambda$ and, thus, the electrical resistance $R_{\mathrm{NW}}$. This phenomenon induces an electrical field below the surface $\left(E_{\mathrm{bar}}\right)$ due to the gas interaction, which is perpendicular to the electrical field applied externally during the conductometric measurement $\left(E_{\text {bias }}\right)$.

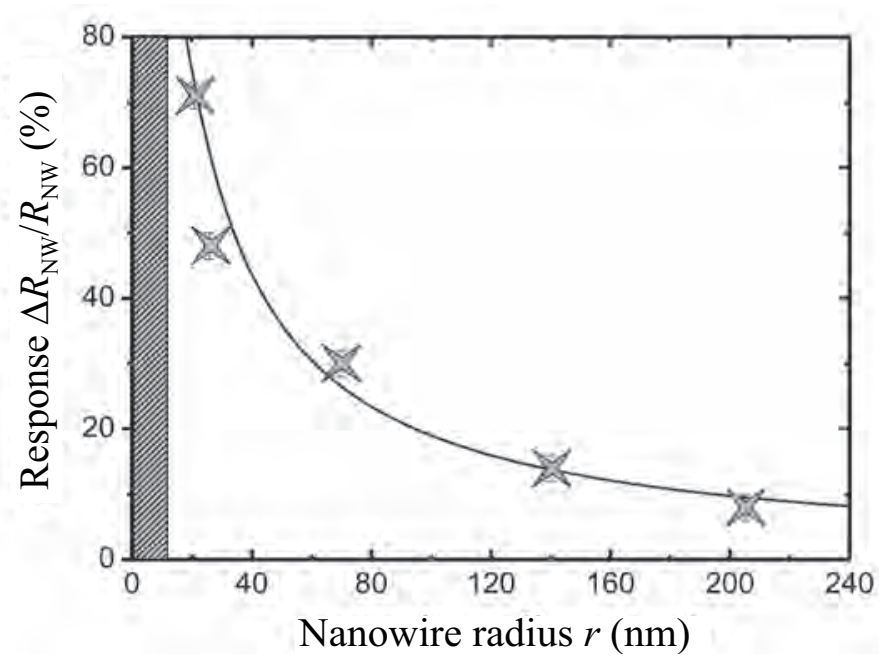

Fig. 3. Response of a $\mathrm{SnO}_{2}$ nanowire to synthetic air/nitrogen pulses as a function of the radius measured at $T \sim 550 \mathrm{~K}$. Higher responses are clearly observed with shrinking dimensions in correspondence with eq. (1) (solid line).

Moreover, the use of catalytic elements to enhance their response $S$ has significantly evolved with extremely good results, as demonstrated elsewhere. ${ }^{(12,19)}$ On the other hand, the first prototypes based on individual nanowires were obtained less than a decade ago. This fact indicates that the testing and validation of their final design remain unfinished, 
and their combination with different catalytic elements and compounds is still in its infancy. ${ }^{(18)}$ Indeed, further research and development is necessary before we are able to compare both technologies in terms of sensitivity, despite the preliminary results obtained to date being very promising. ${ }^{(17)}$

\subsection{Stability}

Single-crystalline metal oxide nanowires with controlled physical, chemical and electrical properties are easily obtained through different synthesis routes, which have been reviewed in detail elsewhere. ${ }^{(1,2,13)}$ These nanomaterials, which are free of nooks and crannies, exhibit high temporal stability as building blocks of gas sensors owing to their well-defined physical structure and atomic layout, since grain boundary effects and gas diffusion are eliminated or at least significantly reduced. Nevertheless, this intrinsic advantage of nanowires is threatened by their size when a probing current $I$ flows through them; the small cross section of nanowires minimizes the effective area that dissipates heat generated by the Joule effect, leading to local increases of temperature $T$, which cause accelerated degradation of the operational life of devices (Fig. 4).(20) Here, it should be pointed out that dissipated power values in the range of a few $\mathrm{mW}$ are sufficiently high to destroy the entire prototype. (20) For this reason, this limitation must be circumvented by selecting suitable experimental conditions, such as applying probing current intensities lower than $I=100 \mathrm{nA}$, which guarantees the device operation for weeks without evidence of degradation. ${ }^{(5)}$ Thus, from the above, one can draw the following conclusion: metal oxide nanowire-based sensors are extremely stable as long as low probing currents are applied. In fact, controlling the self-heating effect in nanowires can be used to design operation strategies capable of significantly reducing the energy consumption of metal oxide sensors, ${ }^{(7,15)}$ avoiding the need for external heaters to rapidly heat them to the temperatures necessary for gas-sensing applications (between 100 and $400^{\circ} \mathrm{C}$ ). Previous works have demonstrated that this experimental approach reduces the thermal inertia of these devices, minimizing the power consumption to only a few tens of $\mu \mathrm{W} .{ }^{(7,15)}$ This is a major breakthrough compared with traditional thin-filmbased sensors.

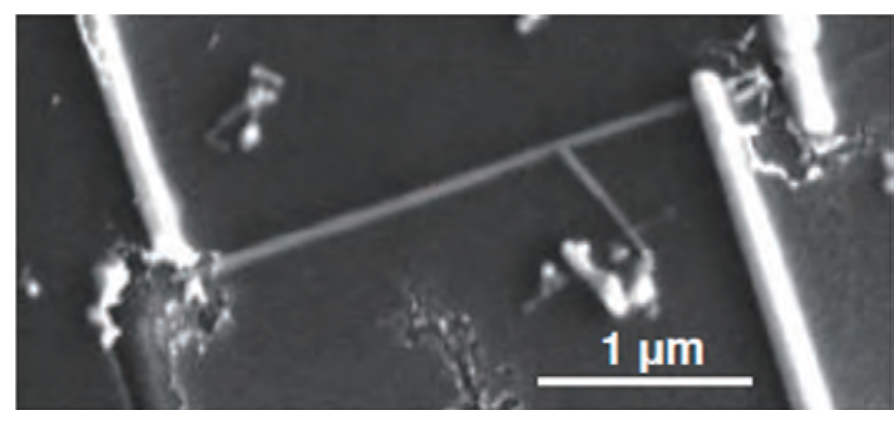

Fig. 4. $\mathrm{SnO}_{2}$ nanowire-based device destroyed after a current peak. A detailed explanation of this process can be found in ref. (20). 


\subsection{Selectivity}

Conductometric metal oxide gas sensors are extensively used in alarm and control applications, in which selectivity towards the interfering gas species is not a critical parameter. In fact, metal oxides have poor selectivity towards different gas species, and the solution to this problem remains a major technological and scientific challenge. ${ }^{(10)}$ This problem is worse in real applications because of the extremely high sensitivity of metal oxides to environmental moisture, which markedly changes both their resistance baseline and the cross-sensitivity to third gases, such as CO. For this reason, much research is being devoted to develop new devices capable of tackling this intrinsic limitation, such as using doped and chemically functionalized sensing layers. . $^{(2,16,21)}$

In theory, metal oxide nanowires suffer the same lack of selectivity towards different gases as thin-film-based devices. Therefore, the above-mentioned discussion is valid for them as well. Some studies try to get around this issue by developing electronic nose (e-nose) systems based on arrays of different metal oxide nanowires, ${ }^{(16)}$ whose responses to gas mixtures are monitored in parallel and recorded. Later, experimental data are electronically analyzed using pattern recognition software, which can be trained to recognize the composition and concentration. On the other hand, it was demonstrated elsewhere that the selectivity of these prototypes is significantly improved by combining controlled illumination conditions ${ }^{(22)}$ and the self-heating effect applied in pulsed mode. ${ }^{(23)}$ Nevertheless, these works are still in their infancy. In conclusion, although promising results were reported in the past, ${ }^{(16)}$ it can be asserted that the lack of selectivity of metal oxide nanowires remains unsolved.

\subsection{Proof-of-concept nanowire-based gas sensors}

For the reasons described thus far in the former sections, ultrathin nanowires $(r<40 \mathrm{~nm})$ are required to obtain optimal gas sensors. To accomplish this objective, the need to fabricate good electrical contacts has become a major priority, and different nanofabrication techniques have been developed in the last few years, such as focusedion-beam (FIB) lithography, ${ }^{(4,15,20,24,25)}$ e-beam lithography, ${ }^{(26)}$ and UV- and shadowmask lithography, ${ }^{(27)}$ enabling the fast engineering of advanced proof-of-concept devices. Their characterization has become extremely useful for determining the sensing properties of different metal oxide nanowires, such as $\mathrm{SnO}_{2}, \mathrm{ZnO}$, and $\operatorname{In}_{2} \mathrm{O}_{3}$, shedding some light on the fundamentals of the gas-surface interaction mechanisms. To date, it has been demonstrated that these sensors exhibit significant and reproducible responses towards oxidizing and reducing gas species (Fig. 5), ${ }^{(14)}$ and some of them operating in self-heated mode require ultralow power consumption to work, particularly if they are compared with metal oxide thin-film-based sensors. Nevertheless, most of these devices were fabricated using the above-mentioned nanolithography techniques, which are only suitable for research prototyping since they are not scalable, and therefore they cannot be applied in industry. ${ }^{(9)}$ For this reason, different alternatives to extend the use of nanowires from the academic level are now under evaluation, such as dielectrophoresis ${ }^{(28,29)}$ and electrospinning. ${ }^{(30)}$ Their combination with portable cost- 
(a)
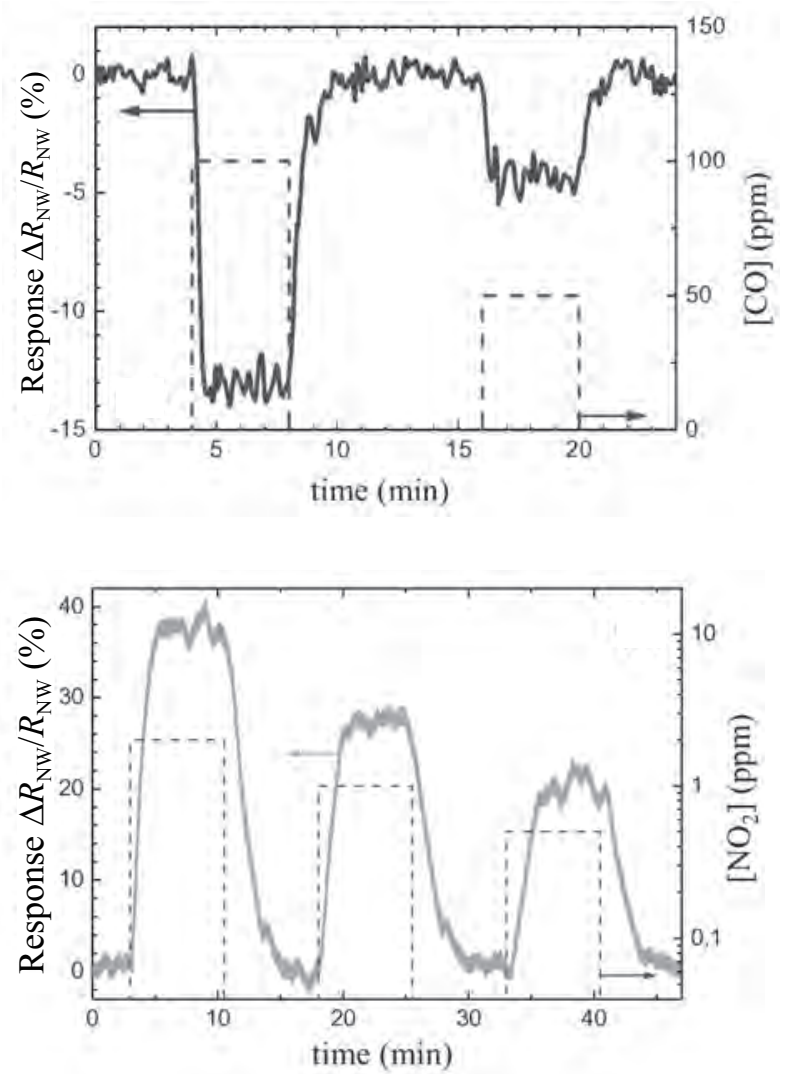

Fig. 5. (a) Response of a $\mathrm{SnO}_{2}$ nanowire towards different $\mathrm{CO}$ pulses at $T=573 \mathrm{~K}$. (b) Response of a $\mathrm{SnO}_{2}$ nanowire towards $\mathrm{NO}_{2}$ pulses at $T=448 \mathrm{~K}$. In both cases, fast and reproducible behaviors are monitored, with excellent recovery of the synthetic air resistance baseline.

effective and consumer-class electronics could pave the way towards a new generation of devices with ultralow power consumption ${ }^{(5,7)}$

\section{Conclusions}

Monocrystalline metal oxide nanowires exhibit unique properties because of their particular morphology and reduced size, making them excellent building blocks of a new generation of gas sensors capable of providing information necessary to elucidate some of the intrinsic phenomena at the nanoscale, such as the interaction mechanisms between gas molecules and the metal oxide semiconductor surfaces. These potential advantages have promoted intensive research efforts to obtain the first proof-of-concept 
devices based on nanowires with good sensitivity, selectivity, and stability towards gases. Although major breakthroughs have been achieved, this technology is still in its infancy. For this reason, further research is required to overcome the unsolved challenge of integrating nanowire gas sensors in low-cost and industrial processes.

\section{Acknowledgments}

This work was partially supported by the Spanish Government [the N-MOSEN (MAT2007-66741-C02-01) and MAGASENS projects] and the EU [the NAWACS (NAN2006-28568-E) project, and the Human Potential Program, Access to Research Infrastructures].

\section{References}

1 M. Law, J. Goldberger and P. Yang: Annu. Rev. Mater. Res. 34 (2004) 83.

2 E. Comini, C. Baratto, G. Faglia, M. Ferroni, A. Vomiero and G. Sberveglieri: Prog. Mater. Sci. 54 (2009) 1.

3 A. Kolmakov and M. Moskovits: Annu. Rev. Mater. Res. 34 (2004) 151.

4 F. Hernandez-Ramirez, A. Tarancon, O. Casals, J. Rodríguez, A. Romano-Rodriguez, J. R. Morante, S. Barth, S. Mathur, T. Y. Choi, D. Poulikakos, V. Callegari and P. M. Nellen: Nanotechnology 17 (2006) 5577.

5 F. Hernandez-Ramirez, J. D. Prades, A. Tarancon, S. Barth, O. Casals, R. Jimenez-Diaz, E. Pellicer, J. Rodríguez, M. A. Juli, A. Romano-Rodriguez, J. R. Morante, S. Mathur, A. Helwig, J. Spannhake and G. Mueller: Nanotechnology 18 (2007) 495501.

6 J. D. Prades, R. Jimenez-Diaz, F. Hernandez-Ramirez, L. Fernandez-Romero, T. Andreu, A. Cirera, A. Romano-Rodriguez, A. Cornet, J. R. Morante, S. Barth and S. Mathur: J. Phys. Chem. C 112 (2008) 14639.

7 J. D. Prades, R. Jimenez-Diaz, F. Hernandez-Ramirez, S. Barth, A. Cirera, A. RomanoRodriguez, S. Mathur and J. R. Morante: Appl. Phys. Lett. 93 (2008) 123110.

8 F. Hernandez-Ramirez, J. Rodriguez, O. Casals, E. Russinyol, A. Vila, A. Romano-Rodriguez, J. R. Morante and M. Abid: Sens. Actuators, B 118 (2006) 198.

9 F. Hernandez-Ramirez et al. (submitted).

10 N. Barsan, D. Koziej and U. Weimar: Sens. Actuators, B 121 (2007) 18.

11 There are many companies offering this type of sensor, such as Figaro, FIS, MICS, UST, CityTech, Applied-Sensors, NewCosmos, etc. See information provided by the manufacturers on their homepages.

(i) http://www.figarosens.com

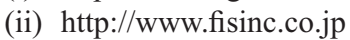

(iii) http://www.appliedsensors.com

(iv) http://www.citytech.com

(v) http://www.microchem.com

12 G. Korotchenkov: Mater. Sci. Eng. R 61 (2008) 1.

13 Z. L. Wang: Materials Today 7 (2004) 26.

14 F. Hernández-Ramírez, A. Tarancón, O. Casals, J. Arbiol, A. Romano-Rodríguez and J. R. Morante: Sens. Actuators, B 121 (2007) 3.

15 E. Strelcov, S. Dmitriev, B. Button, J. Cothren, V. Sysoev and A. Kolmakov: Nanotechnology 19 (2008) 355502. 
16 V. V. Sysoev, B. K. Button, K. Wepsiec, S. Dimitriev and A. Kolmakov: Nano. Lett. 6 (2006) 1584.

17 F. Hernandez-Ramirez, J. D. Prades, A. Tarancon., S. Barth, O. Casals, R. Jimenez-Diaz, E. Pellicer, J. Rodriguez, J. R. Morante, M. A. Juli, S. Mathur and A. Romano-Rodriguez: Adv. Funct. Mater. 18 (2008) 2990.

18 A. Kolmakov, D. O. Klenov, Y. Lilach, S. Stemmer and M. Moskovits: Nano. Lett. 5 (2005) 667.

19 G. Korotcenkov: Sens. Actuators, B 107 (2005) 209.

20 F. Hernandez-Ramirez, A. Tarancon, O. Casals, E. Pellicer, J. Rodríguez, A. RomanoRodriguez, J. R. Morante, S. Barth and S. Mathur: Phys. Rev. B 76 (2007) 085429.

21 Y. Zhang, A. Kolmakov, S. Chretien, H. Metieu and M. Moskovits: Nano. Lett. 4 (2004) 403.

22 J. D. Prades et al: Sens. Actuators, B (in press).

23 J. D. Prades et al. (submitted).

24 C. Y. Nam, D. Tham and J. E. Fischer: Nano. Lett. 5 (2005) 2029.

25 Y.-F. Lin and W.-B. Jian: Nano. Lett. 8 (2008) 3146.

26 D.-W. Kim, I.-S. Hwang, S. J. Kwon, H.-Y. Kang, K.-S. Park, Y.-J. Choi, K.-J. Choi and J.-G. Park: Nano. Lett. 7 (2007) 3041.

27 S. V. Kalinin, J. Shin, S. Jesse, D. Geohegan, P. Baddorf, Y. Lilach, M. Moskovits and A. Kolmakov: J. Appl. Phys. 98 (2005) 044503.

28 S. Kumar, S. Rajaraman, R. A. Gerhardt, Z. L. Wang and P. J. Hesketh: Electrochim. Acta 51 (2005) 943

29 D. Wang, R. Zhu, Z. Zhaoying and X. Ye: Appl. Phys. Lett. 90 (2007) 103110.

30 D. Lin, H. Wu, R. Zhang and W. Pan: Nanotechnology 18 (2007) 465301.

31 S. Mathur, S. Barth, U. Werner, F. Hernandez-Ramirez and A. Romano-Rodriguez: Adv. Mater. 20 (2008) 1550. 